\title{
The Imprint of Lithium Recombination on the Microwave Background Anisotropies
}

\section{Citation}

Zaldarriaga, Matias, and Abraham Loeb. 2002. "The Imprint of Lithium Recombination on the Microwave Background Anisotropies." The Astrophysical Journal 564 (1): 52-59. https:// doi.org/10.1086/324149.

\section{Permanent link}

http://nrs.harvard.edu/urn-3:HUL.InstRepos:41393338

\section{Terms of Use}

This article was downloaded from Harvard University's DASH repository, and is made available under the terms and conditions applicable to Other Posted Material, as set forth at http:// nrs.harvard.edu/urn-3:HUL.InstRepos:dash.current.terms-of-use\#LAA

\section{Share Your Story}

The Harvard community has made this article openly available.

Please share how this access benefits you. Submit a story.

Accessibility 
THE ASTROPHYSICAL JouRNAL, 564:52-59, 2002 January 1

(C) 2002. The American Astronomical Society. All rights reserved. Printed in U.S.A

\title{
THE IMPRINT OF LITHIUM RECOMBINATION ON THE MICROWAVE BACKGROUND ANISOTROPIES
}

\author{
Matias ZaLdARRIAGA ${ }^{1}$ AND ABraham LoEB ${ }^{2}$ \\ Received 2001 May 19; accepted 2001 August 31
}

\begin{abstract}
Following the 2001 paper of Loeb, we explore the imprint of the resonant $6708 \AA$ line opacity of neutral lithium on the temperature and polarization anisotropies of the cosmic microwave background (CMB) at observed wavelengths of 250-350 $\mu \mathrm{m}(0.9-1.2 \mathrm{THz})$. We show that if lithium recombines in the redshift range of $z=400-500$ as expected, then the standard CMB temperature and polarization anisotropies would be significantly modified in this wavelength band. The lithium signal may be difficult to separate from the contamination by the far-infrared background and galactic foregrounds. We show that in polarization, the signal could be comparable to the expected polarization anisotropies of the farinfrared background on subdegree angular scales $(\ell \gtrsim 100)$. Detection of the predicted signal can be used to infer the primordial abundance of lithium, and to probe structure in the universe at $z \sim 500$.

Subject headings: cosmic microwave background - cosmology: theory
\end{abstract}

\section{INTRODUCTION}

The latest measurements of the anisotropies in the cosmic microwave background (CMB; see Halverson et al. 2001; Lee et al. 2001; Netterfield et al. 2001) imply that the days of "precision cosmology" have already arrived. ${ }^{3}$ Future ground- and balloon-based experiments, in combination with the satellite missions Microwave Anisotropy Probe $(M A P)^{4}$ in 2001 and Planck ${ }^{5}$ in 2007, will test current theoretical models to a subpercent precision at photon wavelengths $\gtrsim 500 \mu \mathrm{m}$.

However, at far-infrared wavelengths of $\lesssim 350 \mu \mathrm{m}$, Loeb (2001) has recently shown that the drag force between photons and neutral lithium can strongly modify the CMB anisotropy maps through absorption and reemission at the resonant $6708 \AA$ transition of lithium from the ground state. The drag produced by the hydrogen Ly $\alpha$ line is small, because on the scales of interest, this line is highly optically thick; the hydrogen Balmer lines have a smaller optical depth, but the occupation probability of the lower level is strongly suppressed by the Boltzmann factor. For helium, the transitions correspond to frequencies too far on the Wien tail of the CMB spectrum to be important. Thus, the lithium transition is the most relevant one (Loeb 2001).

Lithium is expected to recombine in the redshift interval $z$ 400-500 (Palla, Galli, \& Silk 1995; Stancil, Lepp, \& Dalgarno 1996, 1998). Despite the exceedingly low lithium abundance ${ }^{6}$ produced in the big bang, the resonant optical depth (Sobolev 1946) after lithium recombination is substantial:

$$
\tau_{\mathrm{Li}}[\lambda(z)]=1.00 f_{\mathrm{Li}_{\mathrm{I}}}(z)\left(\frac{X_{\mathrm{Li}}}{3.8 \times 10^{-10}}\right)\left(\frac{1+z}{500}\right)^{3 / 2},
$$

\footnotetext{
${ }^{1}$ Physics Department, New York University, 4 Washington Place, New York, NY 10003; matiasz@physics.nyu.edu.

${ }^{2}$ Astronomy Department, Harvard University, 60 Garden Street, Cambridge, MA 02138; aloeb@cfa.harvard.edu.

${ }^{3}$ A compilation of all experiments up to date can be found at http:// www.hep.upenn.edu/ $\max / \mathrm{cmb} /$ experiments.html.

${ }^{4}$ Available at http://map.gsfc.nasa.gov.

${ }^{5}$ Available at http://astro.estec.esa.nl/Planck.

${ }^{6}$ Note that by the redshift of interest, all the ${ }^{7}$ Be produced during big bang nucleosynthesis transforms to ${ }^{7} \mathrm{Li}$ through electron capture, since ${ }^{7} \mathrm{Be}$ starts to recombine well before ${ }^{7} \mathrm{Li}$, owing to its significantly higher ionization potential.
}

for an observed wavelength of $\lambda(z)=[(6708 \AA)(1+z)]=$ $(335.4 \mu \mathrm{m})[(1+z) / 500]$. Here, $X_{\mathrm{Li}} \approx 3.8 \times 10^{-10}$ is the latest estimate of the lithium-to-hydrogen number density ratio (Burles, Nollett, \& Turner 2001), and $f_{\mathrm{Li}}(z)$ is the neutral fraction of lithium as a function of redshift (Palla et al. 1995; Stancil et al. 1996, 1998). Loeb (2001) argued that resonant scattering would suppress the original anisotropies by a factor of $\exp \left(-\tau_{\mathrm{Li}}\right)$, but would generate new anisotropies in the CMB temperature and polarization on subdegree scales $(\ell \gtrsim 100)$, primarily through the Doppler effect. Observations at different far-infrared wavelengths could then probe different thin slices of the early universe.

In this paper, we calculate in detail the effect of neutral lithium on both the polarization and temperature anisotropies of the CMB. Section 2 describes the modifications we have made to the standard code CMBFAST (Seljak \& Zaldarriaga 1996) in order to calculate these anisotropies. In $\S 3$ we describe our results and compare them with the foreground noise introduced by the far-infrared emission from galaxies and quasars. Finally, $\S 4$ summarizes the main conclusions of this work.

Throughout the paper, we adopt the low-density cold dark matter (LCDM) cosmological parameters of $\Omega_{\text {cdm }} \approx$ $0.25, \Omega_{\Lambda}=0.7, \Omega_{b}=0.05$, and $H_{0} \approx 70 \mathrm{~km} \mathrm{~s}^{-1} \mathrm{Mpc}^{-1}$, and units of $c=1$.

\section{METHOD OF CALCULATION}

In order to compute the temperature and polarization fluctuations induced by lithium scattering, we complement the standard Thomson opacity $\left(\dot{\tau}_{\mathrm{T}}\right)$ in CMBFAST by a new component $\left(\dot{\tau}_{\mathrm{Li}}\right)$, which is assumed to have a narrow Gaussian shape in conformal time:

$$
\begin{aligned}
\dot{\tau} & =\dot{\tau}_{\mathrm{T}}(\eta)+\dot{\tau}_{\mathrm{Li}}(\eta, v) \\
& =a n_{e} \sigma_{\mathrm{T}}+\tau_{\mathrm{Li}_{\mathrm{I}}} \frac{e^{-\left(\eta-\eta_{\mathrm{Li} \mathrm{I}}\right)^{2} / 2 \sigma_{\eta}^{2}}}{\sqrt{2 \pi \sigma_{\eta}^{2}}},
\end{aligned}
$$

where $\tau$ denotes optical depth, an overdot denotes a conformal time derivative, $a$ is the expansion factor, $\eta$ is the conformal time $(a d \eta=d t), n_{e}$ is the number density of free electrons, and $\sigma_{\mathrm{T}}$ is the Thomson cross section. The lithium opacity depends both on time and observed photon frequency $v$. We characterize this opacity by three parameters: 
the total optical depth $\tau_{\mathrm{Li}}$ given by equation (1), and the central time $\left(\eta_{\mathrm{Li}}\right)$ and width $\left(\sigma_{\eta}\right)$ of the Gaussian peak. The peak is set to a redshift $(1+z)=(\lambda / 6708 \AA)$, where $\lambda=c / v$ is the observed wavelength. The actual (noninstrumental) width of the Gaussian is expected to be $[\Delta z /$ $(1+z)] \sim 3 \times 10^{-5}$ (Loeb 2001). Here we adopt $\sigma_{\eta} / \eta \sim$ $10^{-2}$, as dictated by a fiducial detector's bandwidth.

The lithium opacity introduces a frequency dependence to the CMB anisotropies. Next we show that different frequencies couple only through the net drag force that they provide on the baryons. Our discussion follows the notation of Ma \& Bertschinger (1995).

We consider a density perturbation of comoving wavevector $\boldsymbol{k}$. The photon distribution function can then be expanded to first order in the perturbation amplitude as

$$
\begin{aligned}
f(\boldsymbol{k}, \hat{\boldsymbol{n}}, v, \eta) & =f_{0}(v)[1+\Psi(\boldsymbol{k}, \hat{\boldsymbol{n}}, v, \eta)], \\
\Psi(\boldsymbol{k}, \hat{\boldsymbol{n}}, v, \eta) & =\sum_{l=0}^{\infty}(-i)^{l}(2 l+1) \Psi_{l}(k, v, \eta) P_{l}(\hat{\boldsymbol{k}} \cdot \hat{\boldsymbol{n}}),
\end{aligned}
$$

where $f_{0}(v)=2 / h^{3}\left(e^{h v / k T_{0}}-1\right)$, and where $f$ is a function of the perturbation wavevector $(\boldsymbol{k})$, time $(\eta)$, and the frequency (v) and propagation direction ( $(\hat{n})$ of the photon. In the second line of equation (3), we have expanded the angular dependence of the distribution function in Legendre polynomials.

The distribution function $f(\boldsymbol{k}, \hat{\boldsymbol{n}}, v, \eta)$ satisfies the Boltzmann equation. Since the Thomson cross section is independent of frequency, the standard approach integrates the Boltzmann equation over photon frequencies and uses only one Boltzmann hierarchy to evolve the photon distribution function. In our case, the frequency dependence of the lithium cross section implies that one should solve the hierarchy for different frequencies. Since the frequencies are coupled, one needs to follow all frequencies simultaneously, as done by $\mathrm{Yu}$, Spergel, \& Ostriker (2001) for Rayleigh scattering. The coupling between different frequencies does not originate directly from the scattering term, because lithium scattering does not change the photon frequency. As we show next, the coupling arises from the drag force on the baryons. Binary particle collisions allow the baryons to behave as a single fluid (Loeb 2001) that is subject to the sum of the forces applied by photons at all frequencies. In the limit of no drag force, the different frequencies decouple.

Next, we define the relative density contrast $\left(\delta_{\gamma}\right)$ and velocity divergence of the photon fluid $\left(\theta_{\gamma}\right)$ as

$$
\begin{aligned}
\delta_{\gamma} & =\left(a^{4} \bar{\rho}_{\mathrm{CMB}}\right)^{-1} \int d^{3} v v f_{0}(v) \Psi_{0}(k, v, \eta), \\
\delta \theta_{\gamma}(v) & =\left(a^{4} \bar{\rho}_{\mathrm{CMB}}\right)^{-1} \frac{3}{4} k \Psi_{1}(k, v, \eta), \\
\theta_{\gamma} & =\int d^{3} v v f_{0}(v) \delta \theta_{\gamma}(v) .
\end{aligned}
$$

With these definitions, we can write the equation for the velocity divergence of the baryon fluid $\left(\theta_{b}=k v_{b}\right)$ :

$$
\begin{aligned}
\dot{\theta}_{b}= & -\frac{\dot{a}}{a} \theta_{b}+c_{s}^{2} k^{2} \delta_{b}+\frac{4 \bar{\rho}_{\mathrm{CMB}}}{3 \bar{\rho}_{b}}\left[\dot{\tau}_{\mathrm{T}}\left(\theta_{\gamma}-\theta_{b}\right)\right. \\
& \left.+\int d^{3} v \dot{\tau}_{\mathrm{Li}}(\eta, v) v f_{0}(v)\left(\delta \theta_{\gamma}-\delta \theta_{b}\right)\right], \\
\delta \theta_{b} \equiv & \left(a^{4} \bar{\rho}_{\mathrm{CMB}}\right)^{-1} \theta_{b},
\end{aligned}
$$

where $c_{s}$ is the sound speed of the baryons, $\bar{\rho}_{\mathrm{CMB}}$ and $\bar{\rho}_{b}$ are the mean energy densities of the CMB and the baryons, respectively, and $\delta_{b}$ is the baryon overdensity. The last term in the square brackets of equation (5) is responsible for the coupling between different photon frequencies. However, Figure 1 of Loeb (2001) implies that we can ignore the drag force on the baryons (due to either Thomson or lithium scattering) at the redshifts at which the lithium opacity becomes important $(z \lesssim 500)$. Turning off the Thomson drag (which is larger than the lithium drag) at these redshifts changes the angular power spectrum fluctuations $\left(C_{l}\right.$ 's) by less than $1 \%$, and we expect the error induced by our approximation to be even smaller. Consequently, we can solve the Boltzmann equation separately for each photon frequency by explicitly neglecting the transfer of momentum from the photons to the baryons due to lithium scattering. Even though momentum conservation is not strictly satisfied in this approach, the remaining correction is expected to be negligible.

The last change that we introduce to CMBFAST involves polarization. The cross section for lithium scattering has a different dependence than for Thomson scattering on both scattering angle and Stokes parameters. The scattering matrix for $\left(I_{\|}, I_{\perp}, U\right)$ (in which the parallel and perpendicular directions are defined relative to the scattering plane) can be decomposed into two parts:

$$
\frac{3}{2} E_{1}\left(\begin{array}{ccc}
\cos ^{2} \Theta & 0 & 0 \\
0 & 1 & 0 \\
0 & 0 & \cos \Theta
\end{array}\right)+\frac{1}{2} E_{2}\left(\begin{array}{ccc}
1 & 1 & 0 \\
1 & 1 & 0 \\
0 & 0 & 0
\end{array}\right),
$$

where $\Theta$ is the scattering angle. The first term is the usual Thomson (or Rayleigh) scattering matrix multiplied by a factor $E_{1}$, while the second term, which is proportional to $E_{2}$, does not generate polarization and is isotropic in angle. The two amplitudes depend on the quantum numbers of the resonant states, and satisfy $E_{1}+E_{2}=1$ (Chandrasekhar $1960)$. For the transition between the ground state $(2 S)$ and first excited state $(2 P)$ of lithium, we get $E_{1}=\frac{1}{3}$ (Hamilton 1947; Chandrasekhar 1960). Interference between the $2^{2} S-2^{2} P_{1 / 2}^{0}$ transition and the $2^{2} S-2^{2} P_{3 / 2}^{0}$ transition (Stenflo 1980) has a negligible effect on the polarization. This follows from the fact that $\tau_{\mathrm{Li}_{\mathrm{I}}} \sim 1$, and that for a given lithium atom, a photon will likely scatter when its frequency is separated from the line center by less than the natural width $(37 \mathrm{MHz} / 4 \pi)$, which is much smaller than the frequency separation between these transitions $(10 \mathrm{GHz})$.

\section{RESULTS}

The significance of the new opacity component can be assessed from the visibility function $\Upsilon(\eta)$. This function provides the probability distribution for the time of last scattering of the photons observed today at a conformal time $\eta_{0}$,

$$
\begin{aligned}
& \Upsilon(\eta)=\dot{\tau}(\eta) e^{-\tau(\eta)}, \\
& \tau(\eta)=\int_{\eta}^{\eta_{0}} d \eta^{\prime} \dot{\tau}\left(\eta^{\prime}\right) .
\end{aligned}
$$

In Figure 1, we show the visibility functions for some of the models that we consider later.

The observed anisotropies have two separate contributions, one from the standard last-scattering surface at hydrogen recombination (decoupling), which is suppressed 


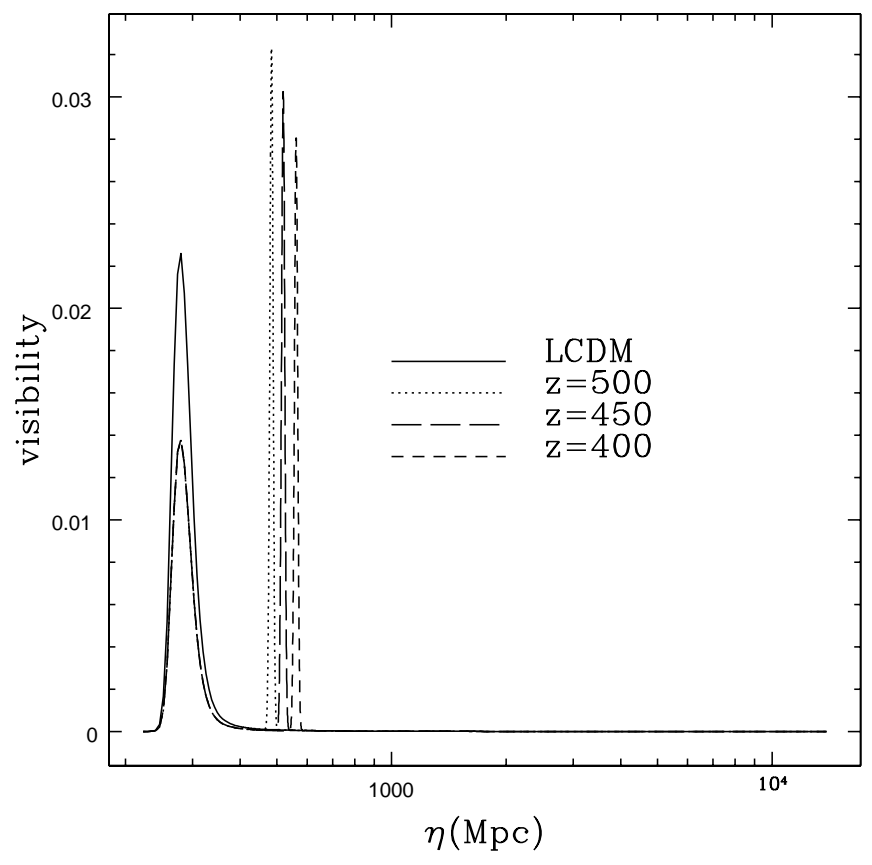

FIG. 1.-Visibility function for models with $\tau_{\text {LiI }}=0.5$ and LCDM. We show three examples, corresponding to resonant scattering at different redshifts: $z=400,450$, and 500. The present-day value of the conformal time is $\eta_{0}=1.39 \times 10^{4} \mathrm{Mpc}$.

by $e^{-\tau_{\mathrm{Li}}}$, and a second new contribution that is generated by lithium scattering at lower redshifts. In the following subsections, we characterize this new contribution to the temperature and polarization anisotropies.

\subsection{Temperature and Polarization Anisotropies}

Figures 2 and 3 show the predicted power spectra for the temperature and polarization anisotropies of the CMB at an observed wavelength of $\lambda=335.4 \mu \mathrm{m}$, corresponding to

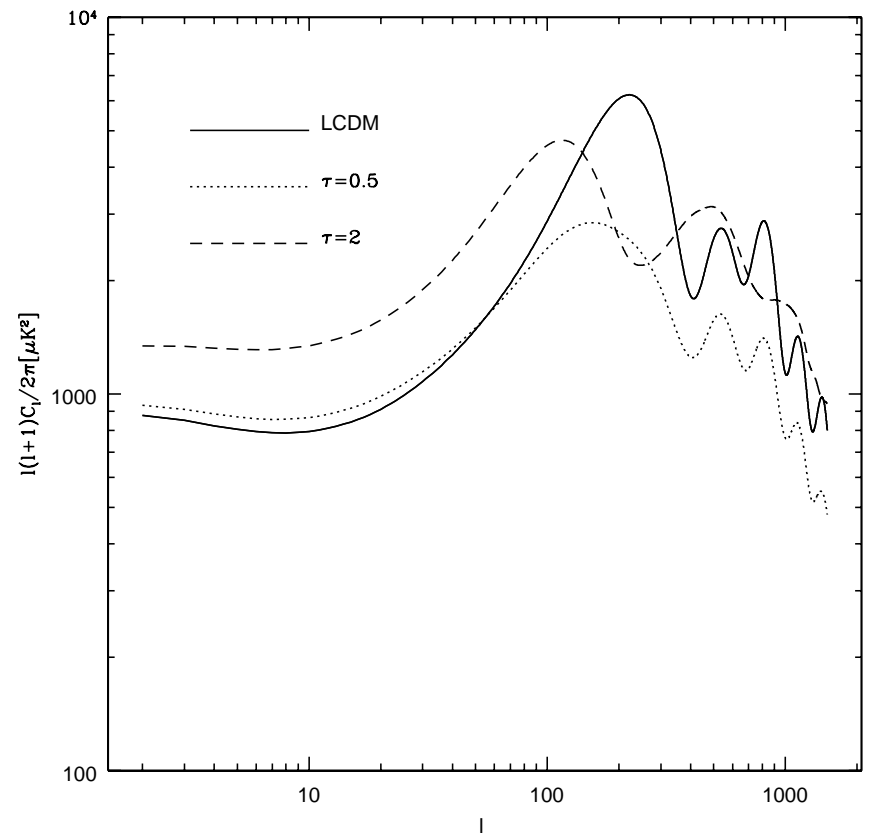

FIG. 2.-Temperature power spectra for the standard calculation (LCDM model) and two other models with lithium optical depths of $\tau_{\mathrm{LiI}}=0.5$ and 2.0 at $z=500$, for observations in a narrow band around $335 \mu \mathrm{m}$. The width of the $\dot{\tau}_{\mathrm{Li} \text { I }}$ Gaussian in eq. (2) is $\sigma_{\eta} / \eta=0.01$.

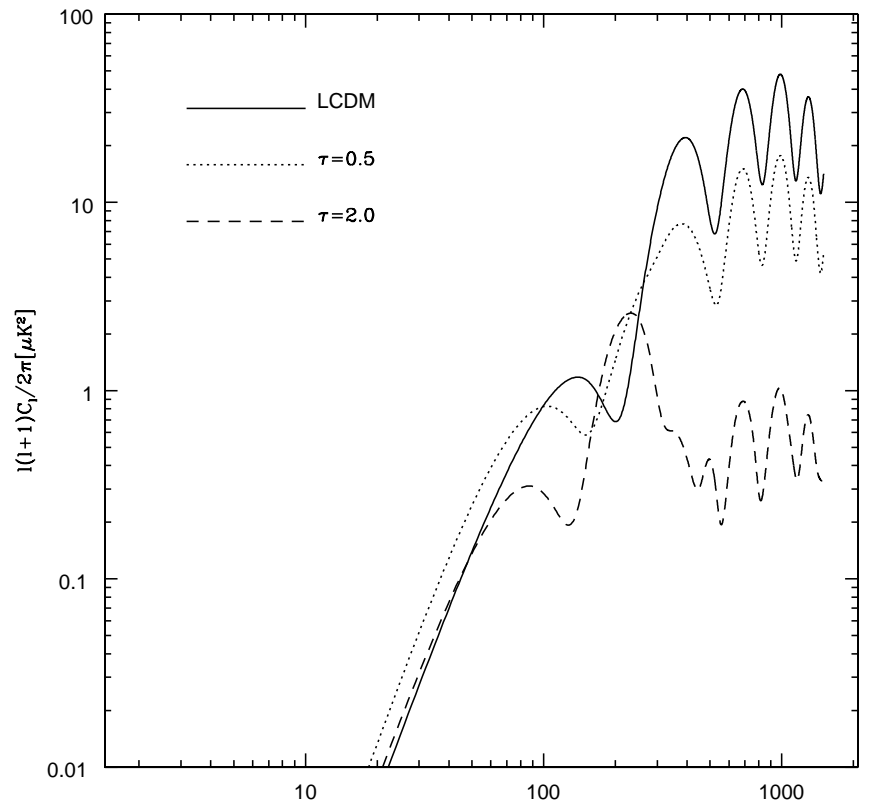

FIG. 3.-Polarization power spectra. Models are the same as in Fig. 2.

lithium scattering at $z=500$. The Stokes parameters are measured in $\mu \mathrm{K}$. The figures compare the spectrum of fluctuations in standard LCDM (no lithium scattering) with two other models, each having a peak in $\dot{\tau}_{\mathrm{Li}}$ at a redshift of $z=500$, but with a total optical depth of either $\tau_{\mathrm{Li} \text { I }}=0.5$ or 2.0. Since at long wavelengths $(\lambda \gtrsim 500 \mu \mathrm{m})$ the LCDM fluctuations are not altered, precise mapping of these fluctuations by the $M A P$ or Planck satellites will provide a reference power spectrum against which the lithium distortion can be measured.

Figure 2 shows that for $\tau_{\mathrm{Li}_{\mathrm{I}}}=0.5$, the small-scale fluctuations are dominated by the suppressed anisotropies from recombination, resulting in a power spectrum that is similar in shape to that of the primary anisotropies, but suppressed in amplitude. However, the $\tau_{\mathrm{Li} \mathrm{I}}=2$ case is very different. Here, the anisotropies are actually larger for many l's than those expected without the lithium scattering, having a different functional dependence on $l$ than the standard case. The $e^{-\tau_{\mathrm{Li} \text { i }}}$ suppression of the original anisotropies is sufficient to make them subdominant relative to the newly generated anisotropies at $z=500$.

There is an interesting difference between the primary anisotropies and those created by lithium scattering. In order to explain it, we introduce the integral solution for the temperature anisotropies,

$$
\begin{aligned}
\left(\frac{\Delta T}{T}+\psi\right)\left(k, \mu, \eta_{0}\right)= & \int_{0}^{\eta_{0}} d \eta\left[\left(\frac{\delta_{\gamma}}{4}+\psi+\mu v_{b}\right) \Upsilon(\eta)\right. \\
& \left.+(\dot{\psi}+\dot{\phi}) e^{-\tau(\eta)}\right] e^{i k \mu\left(\eta-\eta_{0}\right)}
\end{aligned}
$$

where $\mu$ is the cosine of the angle between the wavevector and the direction of observation, and $\phi$ and $\psi$ are the two gravitational potentials defined by the perturbed metric, $d s^{2}=a^{2}(\eta)\left[-(1+2 \psi) d \eta^{2}+(1-2 \phi) d x_{i} d x^{i}\right]$. While the contribution from recombination is dominated by the monopole term $\left(\delta_{\gamma} / 4+\psi\right)$, the lithium anisotropies are dominated by the peculiar velocity term $\left(\propto v_{b}\right)$ on most scales. We illustrate this in Figure 4, in which we show the 


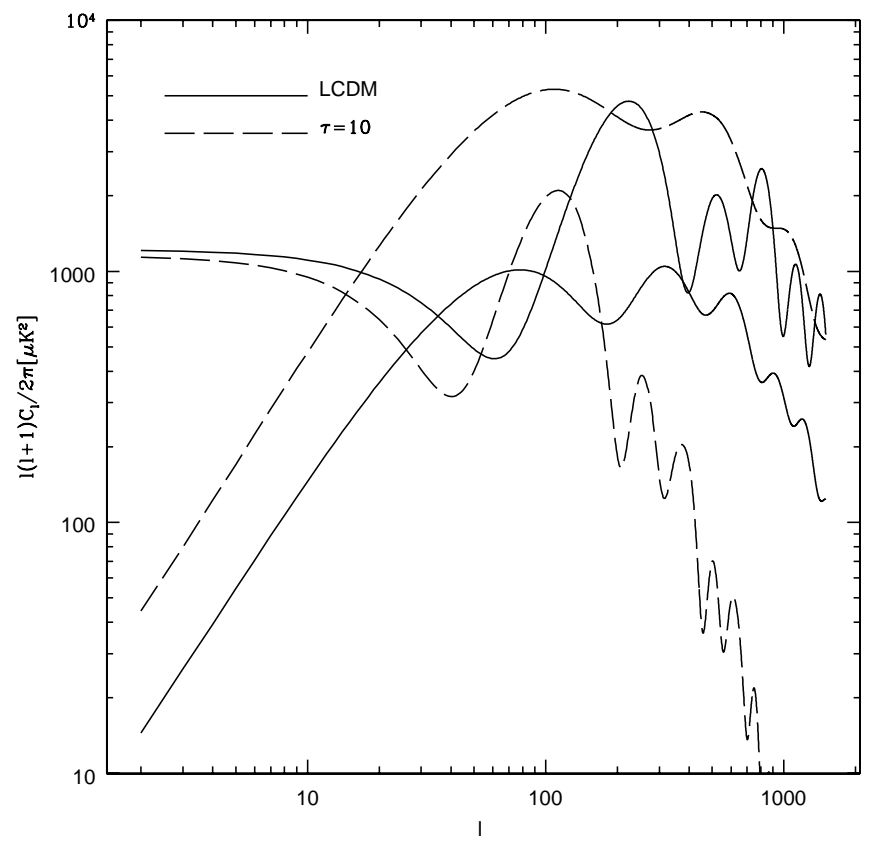

FIG. 4.-Velocity and temperature contribution to anisotropies for LCDM and for a model with $\tau_{\mathrm{Li}_{\mathrm{I}}}=10$ at $z=500$. The curves that approach a finite value at low $l$ are the temperature contributions, and the curves that approach zero at low $l$ describe the velocity contributions.

monopole and velocity contributions to the anisotropies in both the standard LCDM model and in a model that has a large optical depth $\tau_{\mathrm{Li} \mathrm{I}}=10$ at $z=500$. We chose such a large optical depth in order to suppress the original contribution from decoupling. The figure clearly shows that the anisotropies are dominated by the monopole term for the standard LCDM model for almost all values of $l$, while the opposite is true for the $\tau_{\mathrm{Li}}=10$ model. The new anisotropies are dominated by the monopole term only at very low multipoles, $l \lesssim 20$.

We can easily explain why the monopole no longer dominates for the lithium contribution. After recombination, the monopole term decays by the free streaming of the photons, while the velocity of the baryons continues to grow as they fall into dark matter potential wells. For a perturbation mode of wavevector $k$, the monopole term at conformal time $\eta$ after recombination, $\eta>\eta_{\text {rec }}$, is approximately given by

$$
\left(\delta_{\gamma} / 4+\psi\right) \eta=\left(\delta_{\gamma} / 4+\psi\right) \eta_{\mathrm{rec}} j_{0}\left[k\left(\eta-\eta_{\mathrm{rec}}\right)\right],
$$

where $j_{0}(x)$ is the spherical Bessel function. Equation (9) shows that the monopole term is small for $k\left(\eta-\eta_{\mathrm{rec}}\right) \gg 1$ because of the decay in the Bessel function when its argument is large. Figure 1 shows that the new peak of the visibility function occurs at $\eta \sim 500 \mathrm{Mpc}$, while $\eta_{\mathrm{rec}} \sim 300$ $\mathrm{Mpc}$. We can translate the spatial wavenumber $k$ to angular scale using the conformal distance to the new peak in the visibility function, $d=\left(\eta_{0}-\eta\right) \approx \eta_{0}$. We find that $k(\eta$ $\left.-\eta_{\mathrm{rec}}\right) \sim 1.6 \times 10^{-2} l$, which explains why the monopole term is suppressed for $l \gg 60$.

While the monopole term decays between recombination and the lithium-scattering surface, the velocity grows, and thus produces anisotropies that are larger than those generated at decoupling in the $\tau_{\mathrm{Li} \text { I }}=2$ case.

The physics of the polarization anisotropies is different from that of the temperature anisotropies. Since polariza- tion is generated by the quadrupole moment, there are two competing effects that need to be considered. On the one hand, the quadrupole anisotropies are small at recombination, since they are suppressed relative to the velocity fluctuations by a factor $k \delta \eta$, where $\delta \eta$ is the width of the last-scattering surface at recombination (Zaldarriaga \& Harari 1995). In the new scenario, the quadrupole is able to grow during the free-streaming period between recombination and $z \sim 500$. This naturally leads to an increase in the polarization signal. The same effect increases the polarization anisotropies on large scales in models with a substantial optical depth to Thomson scattering after the universe reionizes (Zaldarriaga 1997). On the other hand, due to the nature of resonant-line scattering (Hamilton 1947; Chandrasekhar 1960), only $1 / 3$ of the cross section generates polarization out of this quadrupole, while $2 / 3$ produces unpolarized radiation $\left(E_{1}=1 / 3, E_{2}=2 / 3\right)$. Although the quadrupole is bigger at $z \sim 500$ than at recombination, the newly generated polarization is not as large. For example, Figure 3 shows that even for $\tau_{\mathrm{Li}_{\mathrm{I}}}=2$, the polarization at high multipoles, $l \sim 1000$, is dominated by the suppressed signal from decoupling.

\subsection{Cross-Correlations and Difference Maps}

Let us next consider two temperature anisotropy maps obtained from observations at different wavelengths, $T^{a}(\boldsymbol{\theta})$ and $T^{b}(\theta)$. We define the following spectra in multipole space:

$$
\begin{aligned}
& \left\langle T_{l}^{a} T_{l^{\prime}}^{a}\right\rangle=\delta_{l l^{\prime}} C_{l}^{a a}, \\
& \left\langle T_{l}^{b} T_{l^{\prime}}^{b}\right\rangle=\delta_{l l^{\prime}} C_{l}^{b b}, \\
& \left\langle T_{l}^{a} T_{l^{\prime}}^{b}\right\rangle=\delta_{l l^{\prime}} C_{l}^{a b},
\end{aligned}
$$

with $\delta_{l l^{\prime}}$ being the Kronecker delta. Similar expressions can be written for $E$ - and $B$-type polarization. We then define the cross-correlation coefficient,

$$
R_{l}=\frac{C_{l}^{a b}}{\sqrt{C_{l}^{a a} C_{l}^{b b}}} .
$$

If $R_{l} \rightarrow 1$ across a range of $l$ 's, then the two maps are scaled versions of each other over that range.

Figures 5 and 6 show the correlation coefficients between maps of the anisotropies at different observed wavelengths, labeled by the redshift of the peak in the visibility function. They correspond to the correlation coefficient in three cases: (1) between the primary anisotropies and those measured at $\lambda=335.4 \mu \mathrm{m}(z=500)$, (2) between measurements at $\lambda=335.4$ and $301.8 \mu \mathrm{m}(z=450)$, and (3) between $\lambda=335.4$ and $268.3 \mu \mathrm{m}(z=400)$.

The temperature correlation coefficient between the original map of the primary anisotropies and a map at $335.4 \mu \mathrm{m}$ can be understood as follows. For low $l, R_{l} \approx 1$ because the anisotropies are produced by long wavelength (small $k$ ) modes, and the difference in conformal time between recombination and $z \sim 500$ (which we denote by $d_{\mathrm{rec}-500}$ ) is smaller than the perturbation wavelength, i.e., $\left(k d_{\mathrm{rec}-500} \ll\right.$ 1). The fact that there are two different scattering surfaces does not make a difference for these modes.

On small scales, the situation is more complicated. The component of the anisotropies from decoupling, which is suppressed by a factor $e^{-\tau_{\mathrm{Li}} \text {, }}$, drives the cross-correlation coefficient to unity, because its suppression results merely in rescaling. However, the newly generated anisotropies are uncorrelated with those coming from decoupling if they are 


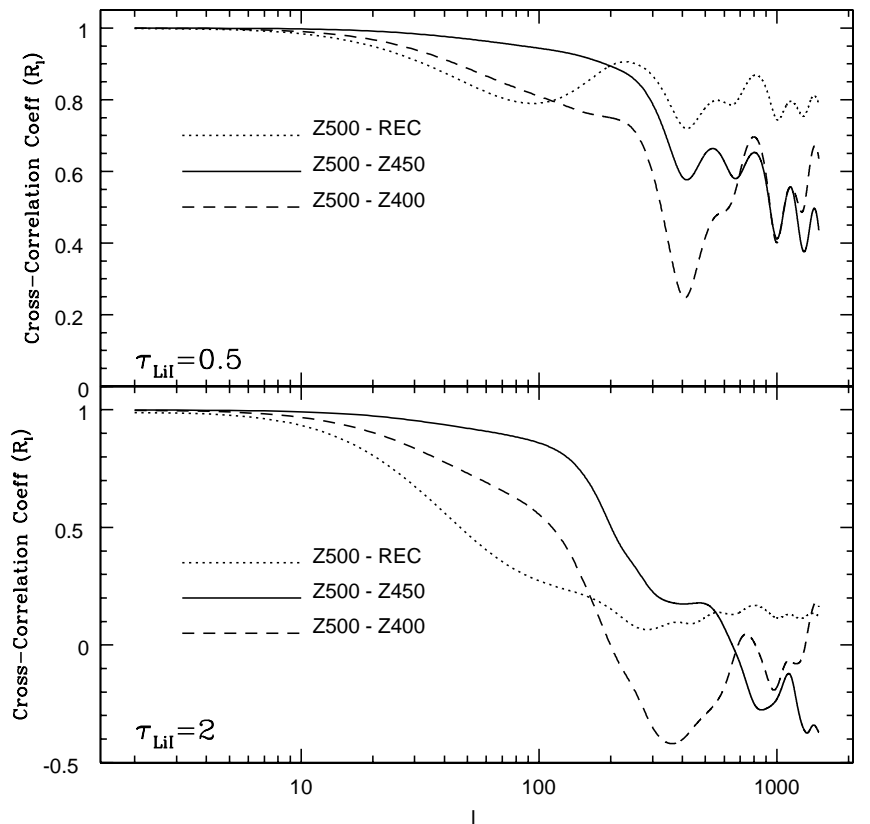

FIG. 5.-Correlation coefficients for temperature anisotropies at three different wavelengths, corresponding to lithium resonances at $z=400$ (268 $\mu \mathrm{m}), 450(302 \mu \mathrm{m})$, and $500(335 \mu \mathrm{m})$. The dotted lines show the correlation coefficient between the standard (long wavelength) anisotropies and those with lithium scattering at $z=500$; the solid line shows the correlation between the anisotropies for 302 and $335 \mu \mathrm{m}(z=500$ and 450); and the dashed lines correspond to 335 and $268 \mu \mathrm{m}(z=500$ and 400). Top: Case of $\tau_{\mathrm{Li} \mathrm{I}_{\mathrm{I}}}=$ 0.5. Bottom: Case of $\tau_{\mathrm{Li} \mathrm{I}}=2.0$.

produced by wavelengths that are smaller than $d_{\mathrm{rec}-500}$, i.e., if $k d_{\mathrm{rec}-500} \sim\left(d_{\mathrm{rec}-500} / d_{\mathrm{rec}}\right) l \sim 1.6 \times 10^{-2} l \gg 1$. The newly generated anisotropies tend to drive the cross-correlation coefficient to zero on small scales.

In Figure 5, we also show the cross-correlations between maps at observed wavelengths for which $\dot{\tau}_{\mathrm{Li}}$ peaks at $z=400,450$, and 500. For a given angular scale, the anisotropies generated by the second peak of the visibility func-

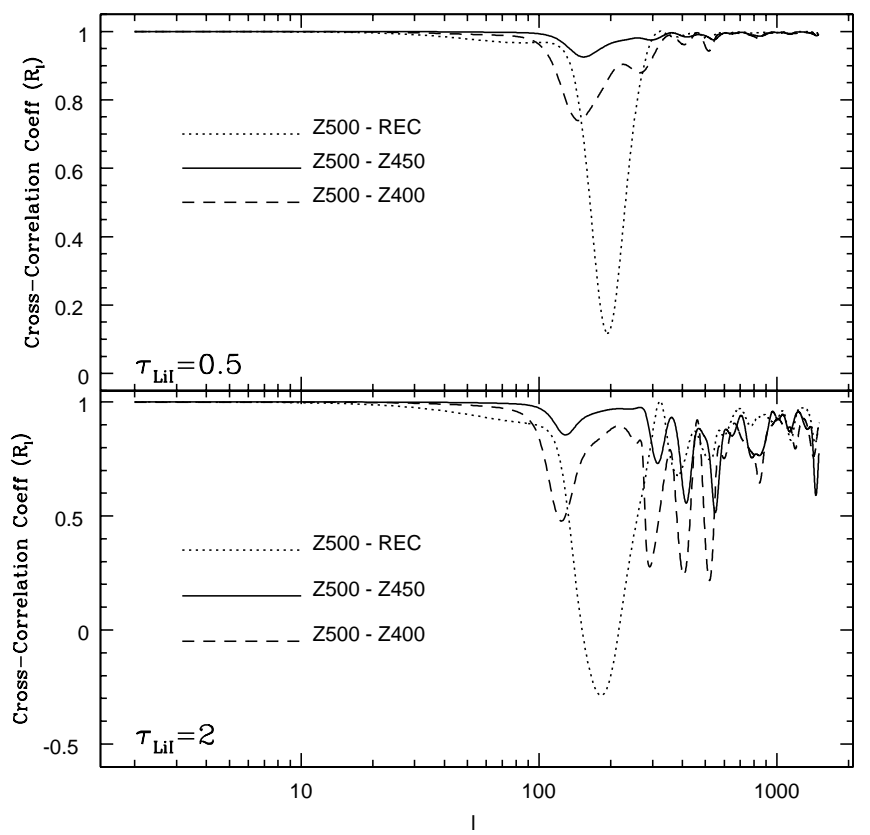

FIG. 6.-Correlation coefficient for polarization. Top: Case of $\tau_{\mathrm{Li}_{\mathrm{I}}}=$ 0.5. Bottom: Case of $\tau_{\mathrm{Li}_{\mathrm{I}}}=2.0$. tion are different only if the conformal distance between the two peaks is much larger than the wavelength of the perturbation producing the anisotropies on that scale. This explains why the departure from $R_{l}=1$ occurs on smaller scales for the correlation between $z=500$ and 450 than for that between $z=500$ and 400 . Also, if the anisotropies in the two maps have a substantial contribution from decoupling, then the correlation coefficient will not approach zero even on small scales, at which the newly generated anisotropies in the two maps are uncorrelated. The significance of this primary contribution from decoupling is suppressed when $\tau_{\mathrm{Li}_{\mathrm{I}}}$ increases, as illustrated by the $\tau_{\mathrm{Li} \mathrm{I}_{\mathrm{I}}}=0.5$ and $\tau_{\mathrm{Li} \text { I }}=2.0$ panels of Figure 5 .

The correlation coefficient for polarization, which is shown in Figure 6, can be explained along similar lines. On small scales, the polarization anisotropies are dominated by the suppressed contribution from recombination, and so $R_{l} \rightarrow 1$. The dominance of the new anisotropies over the primary ones at $l \sim 200$ makes the correlation coefficient between primary and $z=500$ anisotropies deviate away from unity around that scale. However, among neighboring frequencies, the cross-correlation approaches unity near $l \sim 200$, because the distance between the peaks of the visibility function is not sufficient to decorrelate the contributions from the relevant $k$ modes.

In Figures 7 and 8, we show the difference power spectra for two maps at different observed wavelengths. We define $\delta T(\boldsymbol{\theta})=T^{a}(\boldsymbol{\theta})-T^{b}(\boldsymbol{\theta})$ and

$$
\left\langle\delta T_{l} \delta T_{l^{\prime}}\right\rangle=\delta_{l l^{\prime}} C_{l}^{\text {diff }} .
$$

A similar expression can be written for the polarization. The difference spectrum is a measure of the new signature produced by the lithium scattering. The $M A P$ and Planck satellites will measure with high precision the anisotropies at long photon wavelengths, which are not affected by lithium and could be used to construct the difference maps.

The power spectra of the difference maps, shown in Figure 7 for the temperature and Figure 8 for the $E$-type

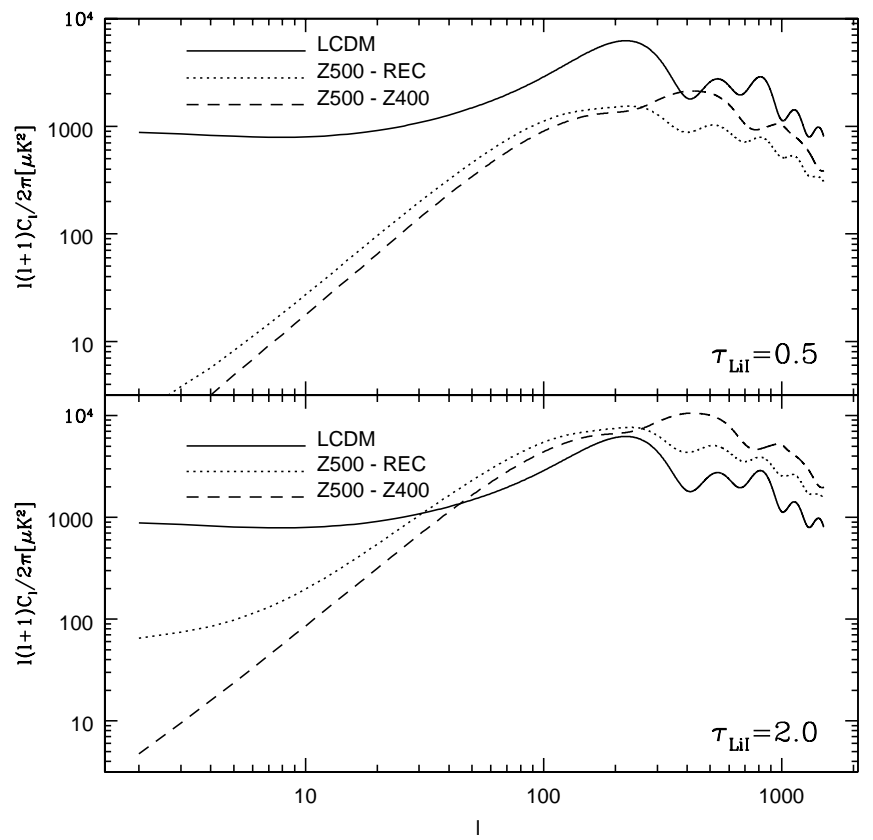

FIG. 7.-Difference power spectra for temperature anisotropies. Top: Case of $\tau_{\mathrm{Li}_{\mathrm{I}}}=0.5$. Bottom: Case of $\tau_{\mathrm{Li}_{\mathrm{I}}}=2.0$. 


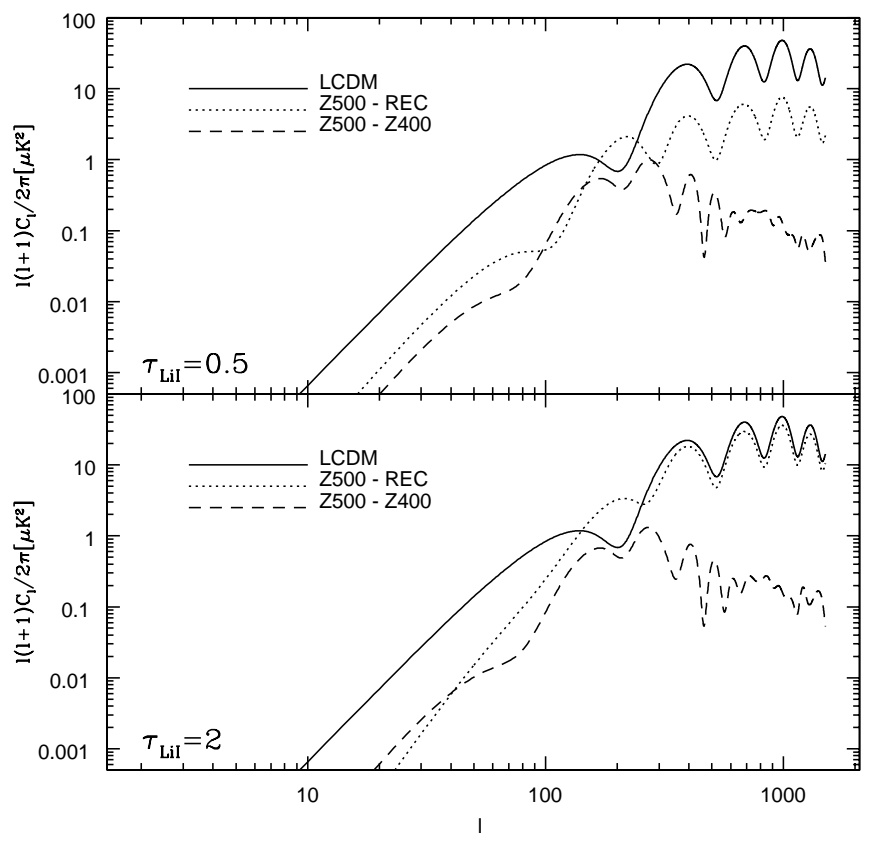

FIG. 8.-Difference spectra for polarization. Top: Case of $\tau_{\mathrm{Li}_{\mathrm{I}}}=0.5$. Bottom: Case of $\tau_{\mathrm{Li} \mathrm{I}}=2.0$.

polarization, are consistent with our interpretation of the cross-correlation spectra between maps. On large scales, the difference between the temperature maps becomes very small. The power in the $Z 500-Z 400$ difference map peaks at $l \sim 450$, while the power for the recombination $-Z 500$ difference peaks at $l \sim 250$. The amplitude of the difference increases as the optical depth increases. When $\tau_{\mathrm{Li} \text { I }}$ is large, the difference spectrum for the recombination $-Z 500$ polarization almost coincides with that of the primary anisotropies, as a result of the fact that the primary anisotropies dominate over the newly generated ones.

\subsection{Comparison with the Far-Infrared Foreground}

The main difficulty in measuring the lithium imprint on the CMB anisotropies is the contamination by the farinfrared background (FIB). There has been no detection to date of the anisotropies in this background, and so we have to rely on theoretical estimates (Haiman \& Knox 2000; Knox et al. 2001).

In order to properly combine the contributions from the CMB and FIB anisotropies, we express intensities in terms of the equivalent Rayleigh-Jeans temperature, $T_{\mathrm{RJ}}$ (in $\mu \mathrm{K}$ ). We start by comparing the temperature anisotropies. The total fluctuation amplitude is given by

$$
\begin{aligned}
\Delta T_{\mathrm{RJ}} & =\Delta T_{\mathrm{RJ}}^{\mathrm{CMB}}+\Delta T_{\mathrm{RJ}}^{\mathrm{FIB}} \\
& =T_{\mathrm{RJ}}^{\mathrm{CMB}}\left(\frac{\Delta T_{\mathrm{RJ}}}{T_{\mathrm{RJ}}}\right)_{\mathrm{CMB}}+T_{\mathrm{RJ}}^{\mathrm{FIB}}\left(\frac{\Delta T_{\mathrm{RJ}}}{T_{\mathrm{RJ}}}\right)_{\mathrm{FIB}} .
\end{aligned}
$$

The ratio between the CMB intensity and the central value for the inferred intensity of the FIB (Fixsen et al. 1998; see the central dot-dashed curve in Fig. 2 of Haiman \& Knox 2000) is of order unity for a lithium-scattering redshift $z \sim 500$ :

$$
\frac{T_{\mathrm{RJ}}^{\mathrm{CMB}}}{T_{\mathrm{RJ}}^{\mathrm{FIB}}} \approx\left(\frac{500}{1+z}\right) \exp \left[15.78\left(1-\frac{500}{1+z}\right)\right] .
$$

As noted by Loeb (2001), the temperature fluctuations in the Wien tail translate to intensity fluctuations $\Delta I_{v}$ (in ergs $\mathrm{s}^{-1} \mathrm{~cm}^{-2} \mathrm{sr}^{-1} \mathrm{~Hz}^{-1}$ ) of a much larger contrast,

$$
\begin{aligned}
\left(\frac{\Delta T_{\mathrm{RJ}}}{T_{\mathrm{RJ}}}\right)_{\mathrm{CMB}} & \equiv \frac{\Delta I_{v}}{I_{v}}=\left(\frac{d \ln I_{v}}{d \ln T}\right) \frac{\Delta T}{T} \\
& =\left(\frac{h v}{k T}\right) \frac{\Delta T}{T}=15.78\left(\frac{500}{1+z}\right) \frac{\Delta T}{T},
\end{aligned}
$$

where we have substituted $I_{v}(T) \propto \exp (-h v / k T)$ and $T=2.725 \mathrm{~K}$ (Mather et al. 1999). The anisotropy amplitude shown in Figure 7 depends on $\tau_{\mathrm{Li}}$, but roughly implies $\left(\Delta T_{\mathrm{RJ}} / T_{\mathrm{RJ}}\right)_{\mathrm{CMB}} \sim 3 \times 10^{-4}[500 /(1+z)]$. Haiman \& $\mathrm{Knox}$ (2000) and Knox et al. (2001) estimate $\left(\Delta T_{\mathrm{RJ}} / T_{\mathrm{RJ}}\right)_{\mathrm{FIB}}=$ 0.05-0.1. The FIB anisotropies peak at an $l$ of a few hundred, but the peak is very broad. The anisotropies in the FIB are relatively large, since they originate from clustering of sources at low redshifts, $z \sim 1$.

We conclude that if $50 \%$ of the lithium ions recombine at $z \sim 500$, then

$$
\begin{aligned}
\frac{\Delta T_{\mathrm{RJ}}^{\mathrm{CMB}}}{\Delta T_{R J}^{\mathrm{FB}} \approx} & 3 \times 10^{-3}\left(\frac{500}{1+z}\right)^{2} \\
& \times \exp \left[15.78\left(1-\frac{500}{1+z}\right)\right] .
\end{aligned}
$$

Since the CMB contribution is subdominant, it is essential to exploit the different frequency dependence of the FIB and CMB anisotropies in order to subtract the FIB contribution with high precision. This might be possible on small angular scales, at which the temperature anisotropies generated by lithium scattering at different redshifts are uncorrelated, as indicated by Figures 5 and 7. Also, since the FIB is produced by point sources, observations with high angular resolution can resolve the sources and remove them individually. Contamination by emission from Galactic dust is also a potential problem. For measurements of the anisotropies in the FIB, it was proposed to look only in the "cleaner" regions of the sky (Knox et al. 2001), which could greatly reduce the contamination. The feasibility of the proposed measurement will only become clear when more is known about the FIB and the sources that produce it. At this stage, it is fair to say that detecting the lithium signal appears to be very difficult.

Next we consider polarization. While the intensity anisotropies of the FIB are dominated by the clustering of the sources, rather than by Poisson fluctuations, the situation is different for the polarization anisotropies. We assume that each source is polarized with a degree of polarization $\epsilon$, and that the polarizations of different sources are uncorrelated. Under these assumptions, it is easy to show that both the $E$ and $B$-polarization spectra share the same amplitude of

$$
\epsilon^{2} / 2 C_{T, l}^{\text {Poisson }},
$$

where $C_{T, l}^{\text {Poisson }}$ is the Poisson contribution to the temperature anisotropies. The Poisson fluctuations can be calculated based on the Submillimeter Common-User Bolometric Array (SCUBA) source counts and are shown as the light solid line in Figure 3 of Haiman \& Knox (2000).

The Poisson component of the FIB anisotropies is approximately a factor of $\sim 100$ smaller in power than the total temperature anisotropies at $l \sim 100-200$. If we adopt an average value of $\epsilon=0.4 \%$ (Jones 1993), then the $E$ polarization is lower by a factor of $(0.004 / \sqrt{2}) 0.1=2.8$ $\times 10^{-4}$ than the intensity fluctuations. 
On the other hand, Figures 2 and 3 imply that the CMB is approximately $10 \%$ polarized for $l \gtrsim 200$. We therefore find that as long as lithium recombines around $z \sim 500$,

$$
\begin{aligned}
\frac{\Delta E_{\mathrm{RJ}}^{\mathrm{CMB}}}{\Delta E_{\mathrm{RJ}}^{\mathrm{FIB}}} \approx & 1.1\left(\frac{\epsilon}{0.004}\right)\left(\frac{500}{1+z}\right)^{2} \\
& \times \exp \left[15.78\left(1-\frac{500}{1+z}\right)\right] .
\end{aligned}
$$

A property that could be very useful in experimental attempts to isolate the signal is that the polarization signature due to lithium has only an $E$-type component, while the FIB polarization has an equal amplitude in both the $E$ and $B$-type components. The $B$-type polarization could therefore be used to monitor the FIB contamination and could play an essential role in the subtraction of the FIB.

Depending on the nature of the sources responsible for the FIB and their luminosity function, it may eventually become possible to resolve most of the FIB through highresolution observations at different wavelengths. This approach is used, for example, in observational studies of the Sunyaev-Zeldovich effect, in which much of the contribution from discrete foreground sources is subtracted out through deep high-resolution observations at either radio or optical-infrared wavelengths. At the present time, there are no available source counts in the wavelength range that we consider here. Closest in wavelength are source counts from the SCUBA instrument (see, e.g., Borys et al. 1999, Fig. 2). If most of the FIB could be resolved, the task of detecting the effect of lithium would become easier, since the overall level of contamination would be drastically reduced. Future studies of the FIB will determine whether this reduction is feasible.

\section{CONCLUSIONS}

We have shown that if more than half of the lithium ions recombine by $z \sim 500$, then the temperature and polarization anisotropies of the CMB would be strongly altered at an observed wavelength of $335 \mu \mathrm{m}$ (see Figs. 2 and 3). For high multipoles, $l \gg 10$, the change is dominated by two contributions: (1) the Doppler anisotropies induced at the sharp lithium-scattering surface; and (2) the uniform $\exp \left(-\tau_{\mathrm{Li}_{1}}\right)$ suppression of the primary anisotropies that were generated at hydrogen recombination (decoupling). Maps taken at wavelengths that are different by only $\sim 10 \%$ are expected to have significant differences (see Figs. 7 and 8 ).

The above signals are superimposed on top of the far infrared background (FIB). Our estimates imply that the lithium imprint on the CMB polarization should be comparable to that provided by the FIB (eq. [18]). Detection is more difficult for the temperature anisotropies (eq. [16]).

The wavelength range that we explored overlaps with the highest frequency channel of the Planck mission $(352 \mu \mathrm{m})$, the Balloon-borne Large-Aperture Sub-millimeter Telescope $^{7}$ (BLAST), which will have 250,350 , and $500 \mu \mathrm{m}$ channels, and the proposed balloon-borne Explorer of Diffuse Galactic Emissions ${ }^{8}$ (EDGE), which will survey 1\% of the sky in 10 wavelength bands between 230 and 2000 $\mu \mathrm{m}$, with a resolution ranging from $6^{\prime}$ to $14^{\prime}$ (see Table 1 in Knox et al. 2001).

In order to optimize the detection of the lithium signature on the CMB anisotropies, a new instrument design is required, with multiple narrow bands $(\Delta \lambda / \lambda \lesssim 0.1)$ at various wavelengths in the range $\lambda=250-350 \mu \mathrm{m}$. The experiment should cover a sufficiently large area of the sky so as to determine reliably the statistics of fluctuations on degree scales. In order to minimize contamination from the FIB, the detector should be sensitive to polarization. For reference, the experiment should also measure the anisotropies at shorter wavelengths, at which the FIB dominates. In order to detect the effect of lithium, high signal-to-noise maps of the primordial CMB should be made for the same region of the sky. Most likely, those maps will become available from future CMB missions such as Planck. A strategy for eliminating the contribution from the brightest FIB sources may also be needed.

The resonant optical depth depends sensitively on the primordial lithium abundance and the recombination history of lithium. More detailed calculations of lithium recombination will be done in a forthcoming paper ( $P$. Stancil et al., in preparation). Detection of the lithium signature will also allow calibration of the primordial lithium abundance, which is a sensitive indicator of the mean value and the clumpiness in the baryon abundance during big bang nucleosynthesis. The lithium abundance in nearby stars is subject to large astrophysical uncertainties (Burles et al. 2001, and references therein). We note that values of the lithium opacity that are higher than the ones we have used are potentially possible. As an extreme example, lithium abundance values as high as $X_{\mathrm{Li}_{\mathrm{I}}} \sim 10^{-8}$ were suggested by models of inhomogeneous big bang nucleosynthesis (Applegate \& Hogan 1985; Sale \& Mathews 1986; Mathews, Alcock, \& Fuller 1990),

The lithium signature on the CMB anisotropies is the only direct probe proposed so far of structure in the universe at a redshift $z \sim 400-500$. This redshift marks the beginning of the "dark ages," which end only after the first generation of galaxies form at $z \sim 20$ (see review by Barkana \& Loeb 2001).

We thank Daniel Eisenstein, David Hogg, and Urŏs Seljak for useful discussions. This work was supported in part by NASA grants NAG 5-7039 and NAG 5-7768, and by NSF grants AST-9900877 and AST-0071019 (for A. L.).

\footnotetext{
${ }^{7}$ Available at http://www.hep.upenn.edu/blast.

${ }^{8}$ Available at http://topweb.gsfc.nasa.gov.
}

\section{REFERENCES}

Applegate, J. H., \& Hogan, C. J. 1985, Phys. Rev. D, 31, 3037 Barkana, R., \& Loeb, A. 2001, Phys. Rep., 349, 125

Borys, C., Chapman, S. C., \& Scott, D. 1999, MNRAS, 308, 527

Burles, S., Nollett, K. M., \& Turner, M. S. 2001, Phys. Rev. D, 63, 3512

Chandrasekhar, S. 1960, Radiative Transfer (New York: Dover)

Fixsen, D. J., Dwek, E., Mather, J. C., Bennett, C. L., \& Shafer, R. A. 1998, ApJ, 508, 123

Haiman, Z., \& Knox, L. 2000, ApJ, 530, 124

Halverson, N. W., et al. 2001, ApJ, submitted (preprint astro-ph/0104489)
Hamilton, D. R. 1947, ApJ, 106, 457

Jones, T. J. 1993, ApJ, 403, 135

Knox, L., Cooray, A., Eisenstein, D., \& Haiman, Z. 2001, ApJ, 550, 7

Lee, A. T., et al. 2001 ApJ, 561, L1

Loeb, A. 2001, ApJ, 555, L1

Ma, C. P., \& Bertschinger, E. 1995, ApJ, 455, 7

Mather, J. C., Fixsen, D. J., Shafer, R. A., Mosier, C., \& Wilkinson, D. T. 1999, ApJ, 512, 511

Mathews, G. J., Alcock, C. R., \& Fuller, G. M. 1990, ApJ, 349, 449 
Netterfield, C. B., et al. 2001, ApJ, submitted (preprint astro-ph/0104460)

Palla, F., Galli, D., \& Silk, J. 1995, ApJ, 451, 44

Sale, K. E., \& Mathews, G. J. 1986, ApJ, 309, L1

Seljak, U., \& Zaldarriaga, M. 1996, ApJ, 469, 437

Sobolev, V. V. 1946, Moving Atmospheres of Stars (Leningrad: Leningrad State Univ.; English transl. 1960, Cambridge: Harvard Univ. Press)

Stancil, P. C., Lepp, S., \& Dalgarno, A. 1996, ApJ, 458, 401 1998, ApJ, 509,1

Stenflo, J. O. 1980, A\&A, 84, 68

Yu, Q., Spergel, D. N., \& Ostriker, J. P. 2001, ApJ, 558, 23

Zaldarriaga, M. 1997, Phys. Rev. D, 55, 1822

Zaldarriaga, M., \& Harari, D. D. 1995, Phys. Rev. D, 52, 3276 